\title{
Phononic Crystals Applied to Localised Surface Haptics
}

\author{
Thomas Daunizeau, David Gueorguiev, Sinan Haliyo and Vincent Hayward
}

\begin{abstract}
Metamaterials are solid lattices with periodicities commensurate with desired wavelengths. Their geometric features can endow the bulk material with unusual properties such as, inter alia, negative indices of refraction or unique absorbing qualities. Mesoscale metamaterials and phononic crystals can be designed to cause the occurence of band gaps in the ultrasonic domain. These localised phenomena induce fixed boundary conditions that correspond to acoustic mirrors which, in turn, can be used to establish waveguides in thin plates. Ultrasonic lubrication has been successfully applied to create haptic interfaces that operate by modulating the apparent friction of a surface. In this study, we demonstrate that phononic crystals can be designed to localise the modulation of friction in specific portions of the surface of a thin plate, opening novel possibilities for the design of surface haptic interfaces.
\end{abstract}

Index Terms-Haptics, ultrasonic lubrication, metamaterials.

\section{INTRODUCTION}

$\mathbf{T}$ HE tactile exploration of surfaces is ubiquitous in daily interactions with devices, whether it be swiping on a smartphone or interacting with a touchpad. Existing devices, however, often lack compelling haptic feedback. As a result, an objective of haptic research is to improve the user experience through the modulation of surface friction [1]. Among other options, attention has recently been given to ultrasonic lubrication as a mean to modulate the apparent friction of a smooth surface [2], [3].

Ultrasonic lubrication, originally employed in industrial applications [4], [5], relies in haptics on ultrasonic transverse waves established in a plate to create a "squeeze film" of air between the skin and the surface [6]. With this technique, the normal load applied by the finger pad onto the surface is shared between the air cushion and the asperities of the stratum corneum of the skin in intimate contact with the surface. The effective contact area is reduced and so is the friction according to mechanisms described in [7], [8].

Since humans are sensitive to frictional changes [9], virtual haptic features can be generated on a smooth surface (e.g. [10], [11]). To date, however, ultrasonic lubrication is limited to global interactions since the standing waves must be established throughout an entire plate. The use of evanescent waves confined in waveguides was proposed to deal with this problem in [12] and [13] but with this approach, localisation is limited to the regions where the actuators are placed. Many applications would benefit from a local control of the frictional properties of surface. For instance, the exploration of textures can involve multiple fingers, each of which is in a different frictional state. Large graphical displays, for

Sorbonne Université, CNRS, Institut des Systèmes Intelligents et de Robotique, ISIR, F-75005 Paris, France

[firstname.name] asorbonne-universite.fr example, could become haptically active only in a region intended to be touched. Another use for localised controlled lubrication could be to minimise power consumption of a haptic interface.

\section{Problem Statement}

The transverse displacement, $w(x, y, t)$ of a standing wave in a plate can be decomposed in spatial and temporal components as in $w(x, y, t)=A(x, y) e^{j(\omega t+\phi)}$. The existence of localised standing waves in a same plate is tied to the existence of boundary conditions. Let us consider two of such waves, $w_{1}(x, y, t)$ and $w_{2}(x, y, t)$, with spatial amplitudes $A_{1}$ and $A_{2}$, existing in two domains, $\Omega_{1}$ and $\Omega_{2}$, as exemplified in Fig. 1 . The continuity of the displacement field at any points $\left(x_{b}, y_{b}\right)$ on a boundary $\mathcal{B}$, implies that $w_{1}\left(x_{b}, y_{b}, t\right)=w_{2}\left(x_{b}, y_{b}, t\right) \forall t$ and therefore that $A_{1}\left(x_{b}, y_{b}\right)=A_{2}\left(x_{b}, y_{b}\right)=0$. In other words, this condition entails that $\mathcal{B}$ must be with zero displacement. It is a boundary where the displacement is imposed, also termed fixed or Dirichlet boundary condition. Physically, such condition means that the impedance of the boundary, $Z_{\mathcal{B}}$, should be large compared to that of the propagating regions, $Z_{\Omega_{1}}$ or $Z_{\Omega_{2}}$. Under this assumption, the transmission coefficients would be $\Gamma_{\Omega_{1}}=\left(2 Z_{\Omega_{1}}\right) /\left(Z_{\Omega_{1}}+Z_{\mathcal{B}}\right) \approx 0$ and $\Gamma_{\Omega_{2}}=\left(2 Z_{\Omega_{2}}\right) /\left(Z_{\Omega_{2}}+Z_{\mathcal{B}}\right) \approx 0$ providing total reflection on each side of the boundary.



Fig. 1. Boundary value problem. Two standing waves meet at an arbitrarily shaped boundary where a Dirichlet condition must apply.

The effective implementation of fixed boundaries is not trivial. Adhesive bonding done with polymeric adhesives would have low impedance in the ultrasonic regime compared to that of the glass or metal plate. The soft mechanical grounding would leak acoustic energy across the boundary and fail to localise waves. Adhesive joints are often an order of magnitude thicker than the targeted wave amplitude which would further prevent them from stopping propagation. 
Advances in "acoustic metamaterials" have given birth to new techniques able to control wave propagation [14]-[16]. Among them, "phononic crystals" have received increasing attention for their ability to stop wave propagation in certain frequency ranges called "band gaps" [17]-[19]. The application of this phenomenon to localise ultrasonic lubrication in surface haptics is investigated here. The general idea is to create boundaries made out of phononic crystals with specific band gaps that coincide with the carrier frequency of a desired standing wave.

\section{Acoustic Metamaterials}

Band gaps are important features of acoustic metamaterials. They correspond to spectral bands within which waves do not propagate. A main design challenge in metamaterials is to tune band gaps to the desired carrier frequencies. For the application at hand, the band gaps should be set between $30 \mathrm{kHz}$ and $225 \mathrm{kHz}$ [20] in thin plates made of materials and dimensions relevant to surface haptics.

\section{A. Origin of Band Gaps}

Band gaps either originate from Bragg scattering (deviation of a wavefront by a lattice) or from local resonances (caused for example by periodic cavities).

Bragg scattering band gaps result from the interference of waves scattered by a lattice of period similar to that of the wavelength. They occur in phononic crystals of size inversely proportional to frequency. This constraint has limited their use to high-frequency applications. An aluminium lattice with a transverse speed of sound, $c=3040 \mathrm{~ms}^{-1}$, and a band gap centred around a carrier frequency, $f=60 \mathrm{kHz}$, would require a spatial period of $c / f \approx 51 \mathrm{~mm}$, which would be impractical.

Local resonance band gaps, also called hybridisation band gaps, rely on the coupling of local resonators to create astonishing effective material properties such as negative elastic moduli and/or negative densities [15]. Periodic arrangements are often preferred to facilitate modelling and manufacture. Sub-wavelength structures are possible, which broadens their scope of application.

\section{B. Choice of Lattice Geometry and Material}

Ultrasonic lubrication calls for two-dimensional lattice periodicity. Planar arrangements derived from Bravais lattices [21] have been proposed: square, rectangular, triangular, hexagonal [22]. Specific arrangements, however, have only a minor influence on locally resonant band gaps. One side of the plate must be flat and free of defects since humans can tactually detect asperities as small as one micron [23]. Thus, geometries such as spiral cuts [24] or slits [25] cannot be conveniently used to alter flexural stiffness locally.

The band structure could also arise from periodic material heterogeneities. An example could be the inclusion of rubbercoated lead balls in an epoxy matrix [26]. The resonators, however, must have high quality factors of at least $Q=100$ which limits the choice of material to aluminium, glass, or other high-stiffness low-dissipation materials.

\section{Candidate Lattice}

The metamaterial is intended to create a band gap that establishes a fixed boundary between ultrasonic standing waves regions. The target carrier was set to about $60 \mathrm{kHz}$, as in many existing surface haptics devices. The square lattice shown in Fig. 2a used pillars to create cantilever beam resonators located at the center of each cell on one side of a plate. Slender pillars, see Fig. 2c, are ideal to design resonators with few parameters. Parsimony makes it convenient to tune the band gaps by numerical optimisation [22]. A monolithic structure can be manufactured at the mesoscale by additive or subtractive manufacturing. The parameters with the least influence on the band gaps were fixed according to manufacturing or functional constraints. Periodicity was set to $a=7 \mathrm{~mm}$, and the thickness was fixed to $e=1.5 \mathrm{~mm}$, to support the loading of a finger and provide standing waves with adequate nodal spacing.
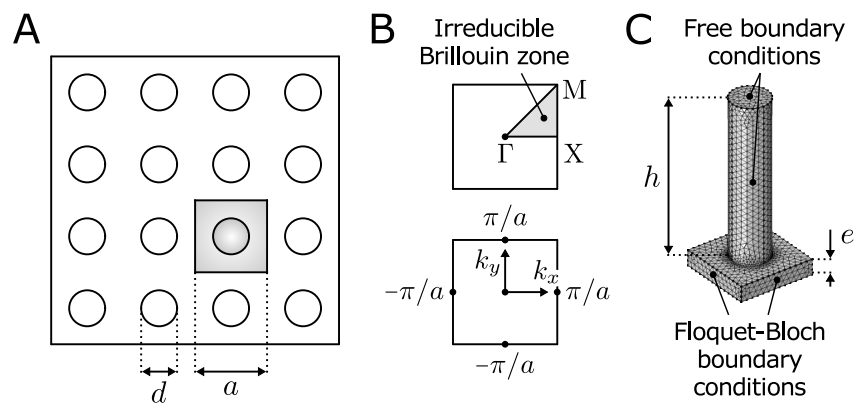

Fig. 2. (A) Top view of a $4 \times 4$ square lattice of cylindrical pillars in the direct space (real space). (B) Irreducible Brillouin zone in the reciprocal space (k-space). (C) Isometric view of a unit cell, meshed with tetrahedral elements for numerical simulations.

\section{Constitutive Equations}

The lattice was to be tuned to possess a band gap at the desired carrier frequency, determined by local resonances. The material was assumed to be linear elastic and to possess homogeneous and isotropic properties. The structure was to satisfy a local dynamic equilibrium given by,

$$
\rho \frac{\partial^{2} \boldsymbol{u}}{\partial t^{2}}=\nabla\left[\mathbf{C}_{\frac{1}{2}}\left(\nabla^{\mathrm{T}} \boldsymbol{u}+\nabla \boldsymbol{u}\right)\right],
$$

where $\rho$ is the density, $\boldsymbol{u}$ is the displacement field, and $\mathbf{C}$ the stiffness tensor. To reveal the band structure, the square lattice was considered to be of infinite periodicity $a$. The Floquet-Bloch's theorem states that the solution of the wave equation is,

$$
\boldsymbol{u}(\boldsymbol{r}+a \boldsymbol{n})=\boldsymbol{u}(\boldsymbol{r}) e^{i a \boldsymbol{k} \cdot \boldsymbol{n}},
$$

where $\boldsymbol{r}=[x, y, z] \in \mathbb{R}^{3}$ is the radius vector, $\boldsymbol{n}=[p q 0] \in$ $\mathbb{Z}^{3}$ is the iteration vector, and $\boldsymbol{k}=\left[k_{x} k_{y} 0\right] \in \mathbb{R}^{3}$ is the wave vector. The wave vector could be restricted to the irreducible Brillouin zone shown in Fig. $2 b$ which is the smallest region within which the problem is devoid of symmetry. This octant region was delimited by three corners, also called "critical points" and marked $\Gamma, \mathrm{X}, \mathrm{M}$. A closed-form solution of these equations was unlikely to be found, so this model served as a basis for numerical simulations. 


\section{NumERicAl Simulations}

\section{A. Implementation}

Several numerical methods have been proposed to solve the constitutive equations and reveal the band structure for this type of systems [27]. To this end, the Finite Element Method (FEM) available in the software Comsol Multiphysics 5.5 was employed. The lattice was assumed to have infinite two-dimensional periodicity, thus the eigenvalue problem could be reduced to that of a unit cell with periodic boundary conditions. A single unit cell was modelled with periodic Floquet-Bloch boundaries applied to the periphery of the base (Fig. 2c). The remaining surfaces were free. Mesh density was increased at the base of the pillar to account for stress concentration. The material properties were: Young's modulus $E=70 \mathrm{GPa}$, Poisson's ratio $\nu=0.33$, and mass density $\rho=2700 \mathrm{~kg} . \mathrm{m}^{-3}$.

\section{B. Dimensional Optimisation}

Dimensionless ratios describing the geometry of the lattice were selected to place the band gap around $60 \mathrm{kHz}$ [22]. The values of the fill ratio, $d / a$, and the aspect ratio, $h / a$, were iterated within ranges of values suitable for manufacturing. At each step, the band gaps were computed to reveal the band structure plotted in Figs. 3a and 3b. Some band gaps were narrow and could result from numerical approximations. The optimal values, $d / a \approx 0.5$ and $h / a \approx 2.1$, corresponded to a band gap that included the ultrasonic carrier and that was wide enough to be immune from manufacturing or material approximations. This band gap ranged from $52.9 \mathrm{kHz}$ to $61.4 \mathrm{kHz}$. The resonator, a cylindrical pillar, could be approximated by a cantilever beam. The flexural modes, $f_{n}$, could be predicted from the Euler-Bernoulli beam theory,

$$
f_{n}=\alpha_{n} \frac{1}{8 \pi} \frac{d}{h^{2}} \sqrt{\frac{E}{\rho}},
$$

where $\alpha_{n}$ is a coefficient associated to the mode of order $n$. The evolution of the resonance frequencies $f_{n}$ was overlaid

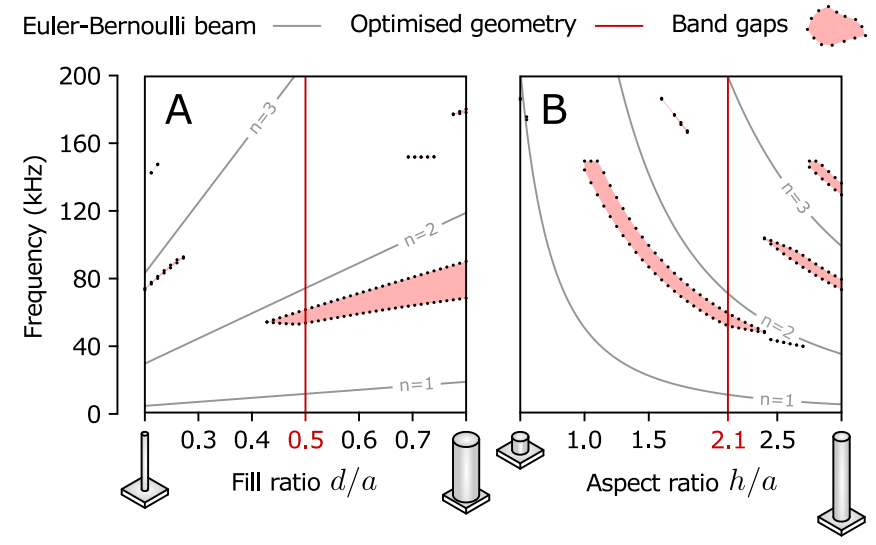

Fig. 3. Evolution of the band gaps in an infinite square lattice of pillars. (A) As a function of the fill ratio $d / a$ with $h / a$ fixed to 2.1. (B) As a function of the aspect ratio $h / a$ with $d / a$ fixed to 0.5 . Black dots represent steps of the FEM simulation.
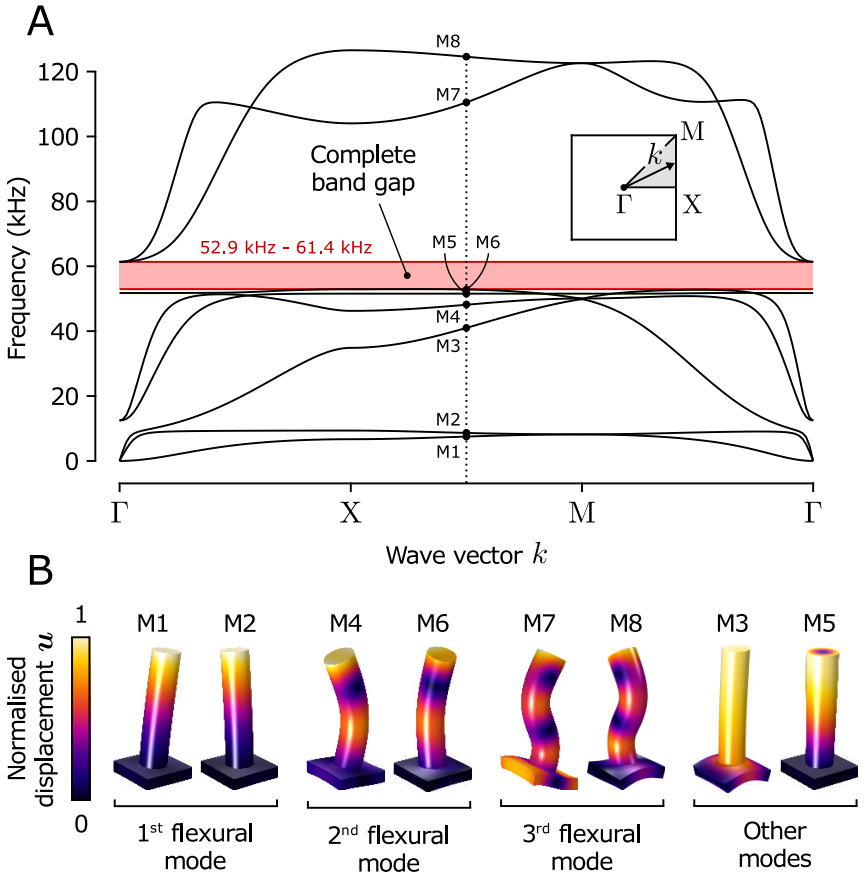

Fig. 4. (A) Dispersion diagram of the optimal lattice for a wave vector spanning the irreducible Brillouin zone. (B) Mode shapes associated to each dispersion curve, captured for a wave vector pointing to the middle of XM as seen on the inset. Displacements are scaled up for visualisation.

with the band structure in Figs. $3 \mathrm{a}$ and $3 \mathrm{~b}$. They both followed similar trends. The frequency increased linearly with the fill ratio, see Fig. 3a, and decreased as an inverse square-law with the aspect ratio, see Fig. 3b. This observation was in agreement with the hypothesis that local resonances were at the origin of the band gaps.

\section{Dispersion}

Dispersion diagrams are key to better understand the physics behind the creation of band gaps. The dispersion diagram of the optimal lattice geometry is given in Fig. 4a. It is obtained by solving the eigenvalue problem for wave vectors sweeping the periphery of the Brillouin zone along $\Gamma \mathrm{X}, \mathrm{XM}, \mathrm{M \Gamma}$. It exhibits several dispersion curves, each corresponding to a normal mode. The diagram reveals the existence of a band gap that extends over the entire Brillouin zone. This case is known as a complete, or absolute, band gap where waves will be totally reflected for any angle of incidence. This property is essential to make good acoustic mirrors in waveguides, where myriads of reflections can give rise to any angle of incidence.

Mode shapes associated with each dispersion curve are illustrated in Fig. $4 \mathrm{~b}$ for a wave vector pointing to the middle of XM. Because of the two-dimensional symmetry, they can be paired according to their flexural mode except for M3 and M5 which are of a different nature, traction and expansion respectively. The upper limit of the band gap was set by the pair M7/M8 while the lower limit was defined by the mutual contributions of M3 / M4 / M5 / M6. This indicated that the band gap in our lattice originated from local resonances. 

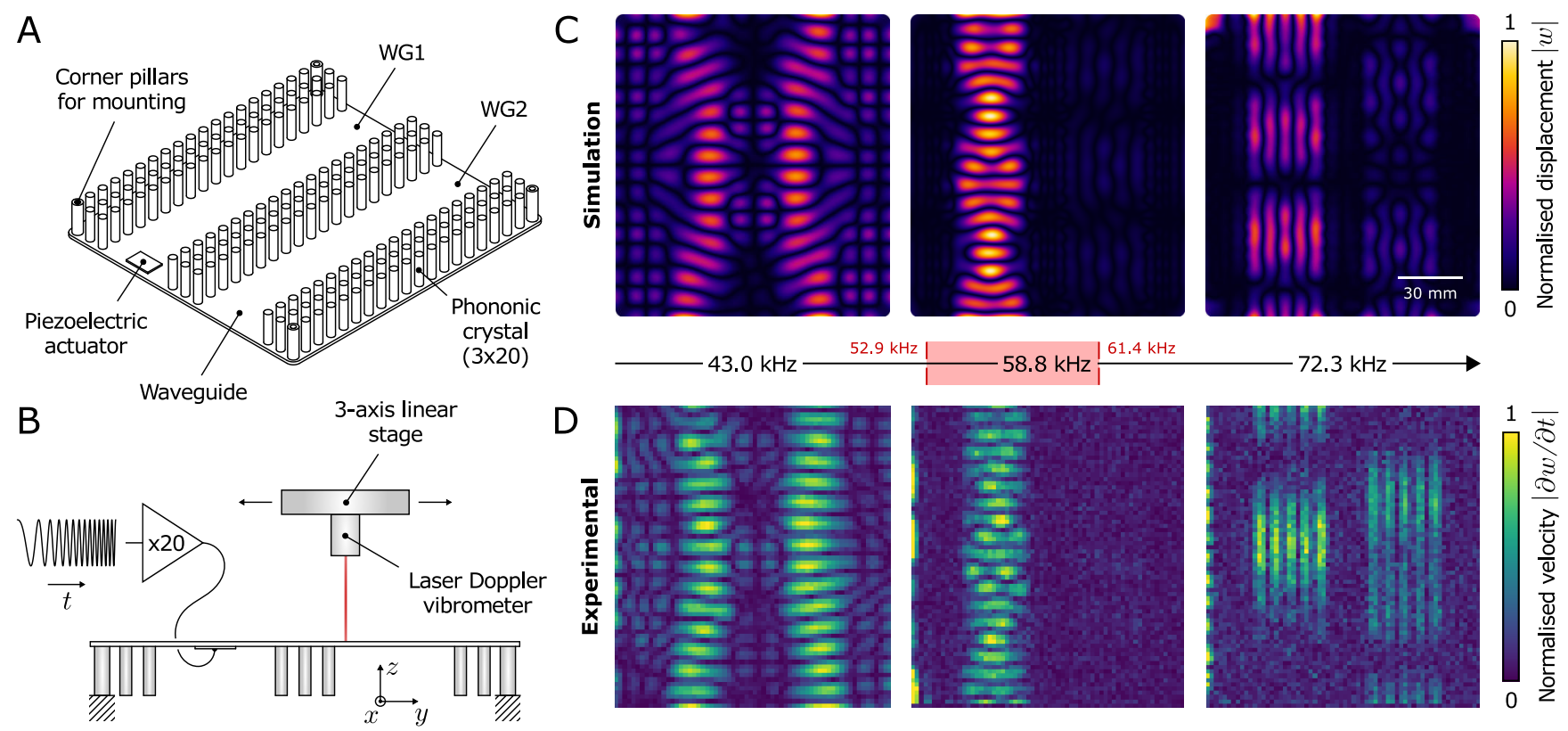

Fig. 5. (A) Schematic of the ultrasonic plate with two independent waveguides WG1 and WG2 (bottom view). (B) Experimental setup with the laser Doppler vibrometer scanning the plate fixed on its four corners (side view). (C) From left to right, numerical simulations of the modal response for three given frequencies respectively, below, inside, and above the band gap (top view). Only transverse displacements $w$ along the z-axis are shown. (D) Optical vibrometry measurements of the modal response for the same frequencies (top view). Velocities along the z-axis are normalised to emphasize mode shapes.

\section{EXPERIMENTAL VALIDATION}

The pillar-based lattice was numerically optimised to provide a complete band gap around the target carrier of $60 \mathrm{kHz}$. This section presents a prototype of an ultrasonic plate using such lattice as a boundary. Its ability to localise standing waves, and consequently friction, was assessed experimentally.

\section{A. Prototype with Two Waveguides}

The lattice was optimised under the assumption of being infinite but a practical implementation is necessarily finite. This affected the lattice performance as it correlates to its number of unit cells. A numerical simulation, not detailed in this paper, has shown that only three layers of unit cells are enough to provide almost total reflection for frequencies in the band gap. The prototype includes two rectangular areas where standing waves could be elicited (Fig. 5a). They are separated by a boundary made out of a phononic crystal with $3 \times 20$ unit cells. This structure was duplicated on the edges since symmetrical boundaries along an ultrasonic plate improve lubrication homogeneity. The two rectangular areas are in essence waveguides and will be further referred as WG1 and WG2. Their dimensions $\left(32 \times 140 \mathrm{~mm}^{2}\right)$ have been selected to constrain a $0 \times 17$ normal mode which corresponds to an ultrasonic standing wave with a nodal spacing of $8 \mathrm{~mm}$ and a resonance frequency $f_{0}=58.8 \mathrm{kHz}$. This value slightly differs from the carrier originally targeted but still falls inside the band gap. The plate was machined out of a single block of aluminium 2017A. Additive techniques like Direct Metal Laser Sintering were prohibited to avoid potential material defects. For our experiments, only the waveguide WG1 needed actuation. The actuator is a piezoelectric plate $\left(11.5 \times 8 \times 0.7 \mathrm{~mm}^{3}\right)$ made out of SM111 piezoceramic material (Steiner \& Martins). It was bonded to the aluminium plate with conductive epoxy ( 8331 , MG Chemicals) to provide an electrical path to the bottom electrode.

\section{B. Localised Ultrasonic Standing Waves}

In order to assess standing waves localisation, we measured the modal response as a function of both space and frequency. To that extent, out-of-plane vibrations of the ultrasonic plate were recorded with a laser Doppler vibrometer (OFV-5000 and MLV-100, Polytec). The sensing head was mounted on a 3-axis linear stage that scanned the entire surface with lateral steps of $\Delta x, y=2 \mathrm{~mm}$ (see Fig. 5b). For each point, a chirp from DC to $100 \mathrm{kHz}$ drove the piezoelectric actuator. This signal was generated at a rate of $200 \mathrm{kHz}$ by a 16-bit acquisition card (NI6343, National Instrument) and fed to a high-power operational amplifier (A400DI, FLC Electronics). A conservative amplitude of $\pm 60 \mathrm{~V}$ was applied to the piezoelectric element to prevent saturation of the amplifier.

For comparison, results from numerical simulations in Fig. 5c are given along with experimental results in Fig. 5d. This ultrasonic plate has a high modal density but for the sake of clarity, only three modes are illustrated. They are selected below, inside and above the band gap. As expected, when actuated at a frequency inside the band gap, only the waveguide WG1 vibrates. The high contrast with the rest of the plate underlines the efficiency of the 3-layer lattice to reflect waves in the band gap. Below the band gap, vibrations spread over the entire plate as if there was no 
boundary. In that case, the vibration amplitude is lower at the lattice location than in the waveguides. This likely occurs because of the added mass of the cylindrical pillars. Similar conclusions can be drawn for vibrations above the band gap.

Our pillar-based metamaterial localises ultrasonic waves. However, it remains to be shown that these waves induce localised lubrication. In that regard, tactile friction measurements were carried out.

\section{Setup for Friction Measurements}

The ultrasonic plate was mounted on a 6-axis force/torque sensor (Nano17, ATI Industrial Automation), see Fig. 6b. Bandwidth drop due to the added mass of the apparatus was not an issue since only low-frequency friction was of interest. Signals were digitised at $1 \mathrm{kHz}$ by a 16-bit acquisition card (NI6343, National Instrument) followed by a zero-lag, 2-pole Butterworth low-pass filter with a $50 \mathrm{~Hz}$ cutoff. Tactile interactions were measured with a noise floor of $21 \mathrm{mN}$ RMS. A sinusoidal carrier at $58.8 \mathrm{kHz}$ with amplitude $\pm 200 \mathrm{~V}$ was fed to the piezoelectric actuator to force the localised mode seen in Fig. 5d.

\section{Methods}

Four different frictional conditions are necessary to conclude on the problem of localised lubrication. They come from the combination of two binary variables: the piezoelectric actuation (turned ON or OFF) and the waveguide tactually explored (either WG1 or WG2). They are marked $\mathrm{C} 1$ to $\mathrm{C} 4$ (Fig. 6c). Skin frictional properties could change over time due to moisture occlusion. To minimize this effect, these conditions were presented in alternate order. The experiment was thus divided in 8 blocks of 30 trials each. Even blocks consisted of tactile explorations alternating between conditions $\mathrm{C} 1$ and $\mathrm{C} 3$, whereas odd blocks alternated between conditions $\mathrm{C} 2$ and $\mathrm{C} 4$. Overall, the four conditions $\mathrm{C} 1$ to $\mathrm{C} 4$ were repeated 60 times per participant. Three volunteers (one male, two females) aged 26 on average gave their written informed consent to participate in this study. They were asked to perform tactile explorations with their dominant index finger in a single stroke in the ulnar direction as shown in Fig. 6a. Visual markers indicated the centreline of the waveguide they had to slide along and a metronome gave audible cues to help them maintain a consistent sliding velocity of $78 \pm 13 \mathrm{~mm} . \mathrm{s}^{-1}$ (mean $\pm \sigma$ ). The normal force was kept around $0.8 \pm 0.4 \mathrm{~N}$ throughout the experiment. Participants washed their hands before the experiment and the ultrasonic plate was cleaned between each block with isopropyl alcohol to remove residual sweat.

\section{E. Results}

The coefficient of kinetic friction $\mu_{k}$, defined during motion as $F_{y} / F_{z}$, was averaged over 60 trials for each condition. The resulting arithmetic means $\left\langle\mu_{k}\right\rangle$ are presented in Fig. 6d for the three participants labelled S1, S2 and S3. The important variance for participant S2 could possibly arise from a larger amount of sweating that caused important variability of the coefficient of friction [28].
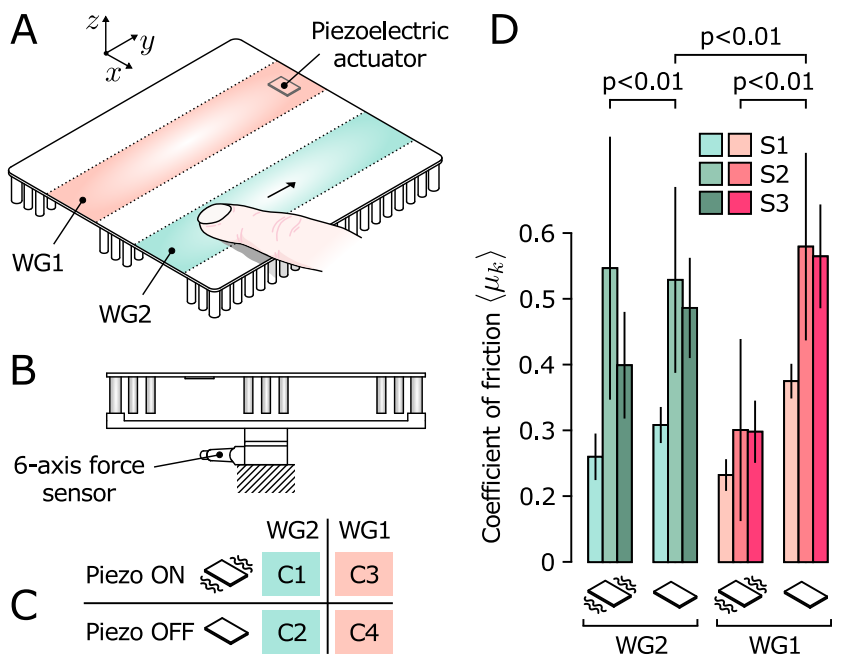

Fig. 6. (A) Illustration of the experiment where participants slid their dominant index finger along the waveguides. (B) Assembly with the force/torque sensor (side view). (C) Table summing the four possible conditions. (D) Mean coefficients of friction for each condition and each participant. Error bars represent one standard deviation.

Conditions with the actuator turned off provide a baseline value of friction. In that case, the mean coefficients of friction are similar for both waveguides since the plate is made from a single block of material. Upon closer inspection, friction is actually slightly higher for WG1, likely because the manufacturing left an inhomogeneous surface finish.

On the one hand, conditions involving WG1 reveal a significant reduction of friction of 55\% across participants when the actuator is turned on (paired t-test with Bonferroni correction, $\mathrm{p}<0.01,95 \%$ CI $[-0.25,-0.21])$. This demonstrates that lubrication is created inside this waveguide similarly as in existing ultrasonic devices.

On the other hand, conditions involving WG2 show a minor reduction of friction of only $11 \%$ when the actuator is turned on (paired t-test with Bonferroni correction, $\mathrm{p}<0.01$, $95 \%$ CI $[-0.06,-0.02])$. This corresponds to a small energy leakage from WG1 towards WG2 as predicted by numerical simulations and laser measurements (middle Figs. 5c and 5d). Despite this leakage, about $80 \%$ of the friction reduction in WG1 is stopped by the 3-layer lattice. Our pillar-based metamaterial therefore effectively localises ultrasonic lubrication.

\section{CONCLUSION}

This paper introduced a method to localise ultrasonic lubrication in thin plates. This novel approach takes advantage of the remarkable properties of acoustic metamaterials and phononic crystals. These engineered periodic structures feature frequency ranges, called band gaps, in which waves do not propagate. In essence, the proposed strategy is to tune these band gaps to the desired ultrasonic carrier frequency. Accordingly, phononic crystals create artificial fixed boundaries that behave like mirrors. These boundaries can be arranged spatially to create waveguides. Guidelines were provided to assist the design of locally resonant phononic crystals relevant to surface haptics. Both laser vibrometry and 
friction measurements gave experimental evidences of their efficiency. With the current implementation, stimuli cannot be delivered on the whole surface since the center and edges are virtually fixed. Ongoing studies aim at scaling down the unit cell to reduce these dead zones. Future works will also investigate the effects of artificial fixed boundaries on the quality factor.

Existing ultrasonic devices can only modulate friction over time while the prototype described in this paper modulates friction both in time and space. This capability opens the door to new multi-touch haptic interactions. More generally, the simplicity and effectiveness of this solution enable numerous potential applications, not only restricted to surfaces. For instance, acoustic metamaterials could be leveraged to make lenses that would provide mid-air haptics with resolutions beyond the diffraction limit.

\section{ACKNOWLEDGMENT}

The authors would like to thank Charles Hudin for its invaluable assistance regarding measurements with the laser Doppler vibrometer.

\section{REFERENCES}

[1] V. Levesque, L. Oram, K. MacLean, A. Cockburn, N. D. Marchuk, D. Johnson, J. E. Colgate, and M. A. Peshkin, "Enhancing physicality in touch interaction with programmable friction," in Proceedings of the SIGCHI Conference on Human Factors in Computing Systems, 2011, pp. 2481-2490.

[2] M. Biet, F. Giraud, and B. Lemaire-Semail, "Squeeze film effect for the design of an ultrasonic tactile plate," IEEE Transactions on Ultrasonics, Ferroelectrics and Frequency Control, vol. 54, no. 12, pp. 2678-2688, 2007.

[3] L. Winfield, J. Glassmire, J. E. Colgate, and M. Peshkin, "T-pad: Tactile pattern display through variable friction reduction," in Second Joint EuroHaptics Conference and Symposium on Haptic Interfaces for Virtual Environment and Teleoperator Systems. IEEE, 2007, pp. 421-426.

[4] H. D. Fridman and P. Levesque, "Reduction of static friction by sonic vibrations," Journal of applied physics, vol. 30, no. 10, pp. 1572-1575, 1959.

[5] A. G. Rozner, "Effect of ultrasonic vibration on coefficient of friction during strip drawing," The Journal of the Acoustical Society of America, vol. 49, no. 5A, pp. 1368-1371, 1971.

[6] E. O. J. Salbu, "Compressible Squeeze Films and Squeeze Bearings," Journal of Basic Engineering, vol. 86, no. 2, pp. 355-364, 061964.

[7] M. Wiertlewski, R. Fenton Friesen, and J. E. Colgate, "Partial squeeze film levitation modulates fingertip friction," Proceedings of the $\mathrm{Na}$ tional Academy of Sciences, vol. 113, no. 33, pp. 9210-9215, 2016.

[8] E. Vezzoli, Z. Vidrih, V. Giamundo, B. Lemaire-Semail, F. Giraud, T. Rodic, D. Peric, and M. Adams, "Friction reduction through ultrasonic vibration part 1: Modelling intermittent contact," IEEE transactions on haptics, vol. 10, no. 2, pp. 196-207, 2017.

[9] D. Gueorguiev, E. Vezzoli, A. Mouraux, B. Lemaire-Semail, and J.L. Thonnard, "The tactile perception of transient changes in friction," Journal of The Royal Society Interface, vol. 14, no. 137, p. 20170641, 2017.

[10] J. Monnoyer, E. Diaz, C. Bourdin, and M. Wiertlewski, "Ultrasonic friction modulation while pressing induces a tactile feedback," in International Conference on Human Haptic Sensing and Touch Enabled Computer Applications (EuroHaptics 2016). Springer, 2016, pp. 171179.

[11] W. B. Messaoud, M.-A. Bueno, and B. Lemaire-Semail, "Textile fabrics' texture: from multi-level feature extraction to tactile simulation," in International Conference on Human Haptic Sensing and Touch Enabled Computer Applications. Springer, 2016, pp. 294-303.

[12] C. Hudin, "Local friction modulation using non-radiating ultrasonic vibrations," in 2017 IEEE World Haptics Conference (WHC). IEEE, 2017, pp. 19-24.
[13] A. B. Dhiab and C. Hudin, "Confinement of vibrotactile stimuli in narrow plates," in IEEE World Haptics Conference. Tokyo, Japan: IEEE, 2019, pp. 431-436.

[14] H. Chen and C. T. Chan, "Acoustic cloaking in three dimensions using acoustic metamaterials," Applied Physics Letters, vol. 91, no. 18, p. 183518, Oct. 2007.

[15] Z. Yang, J. Mei, M. Yang, N. H. Chan, and P. Sheng, "MembraneType Acoustic Metamaterial with Negative Dynamic Mass," Physical Review Letters, vol. 101, no. 20, p. 204301, Nov. 2008.

[16] F. Lemoult, M. Fink, and G. Lerosey, "Acoustic Resonators for FarField Control of Sound on a Subwavelength Scale," Physical Review Letters, vol. 107, no. 6, p. 064301, Aug. 2011.

[17] M. S. Kushwaha, P. Halevi, L. Dobrzynski, and B. Djafari-Rouhani, "Acoustic band structure of periodic elastic composites," Physical Review Letters, vol. 71, no. 13, pp. 2022-2025, Sep. 1993.

[18] R. Martínez-Sala, J. Sancho, J. V. Sánchez, V. Gómez, J. Llinares, and F. Meseguer, "Sound attenuation by sculpture," Nature, vol. 378, no. 6554, pp. 241-241, Nov. 1995.

[19] F. R. Montero de Espinosa, E. Jiménez, and M. Torres, "Ultrasonic Band Gap in a Periodic Two-Dimensional Composite," Physical Review Letters, vol. 80, no. 6, pp. 1208-1211, Feb. 1998.

[20] F. Giraud, T. Hara, C. Giraud-Audine, M. Amberg, B. LemaireSemail, and M. Takasaki, "Evaluation of a friction reduction based haptic surface at high frequency," in 2018 IEEE Haptics Symposium (HAPTICS). San Francisco, CA: IEEE, Mar. 2018, pp. 210-215.

[21] A. Bravais, "Mémoire sur les systèmes formés par les points distribués régulièrement sur un plan ou dans l'espace," Journal de l'École Polytechnique, vol. 19, pp. 1-128, 1850.

[22] R. Pourabolghasem, A. Khelif, S. Mohammadi, A. A. Eftekhar, and A. Adibi, "Physics of band-gap formation and its evolution in the pillar-based phononic crystal structures," Journal of Applied Physics, vol. 116, no. 1, p. 013514, 2014.

[23] R. H. LaMotte and J. Whitehouse, "Tactile detection of a dot on a smooth surface: peripheral neural events," Journal of Neurophysiology, vol. 56, no. 4, pp. 1109-1128, Oct. 1986.

[24] A. Foehr, O. R. Bilal, S. D. Huber, and C. Daraio, "Spiral-based phononic plates: From wave beaming to topological insulators," Phys. Rev. Lett., vol. 120, p. 205501, May 2018.

[25] O. R. Bilal and M. I. Hussein, "Trampoline metamaterial: Local resonance enhancement by springboards," Applied Physics Letters, vol. 103, no. 11, p. 111901, Sep. 2013.

[26] Z. Liu, "Locally resonant sonic materials," Science, vol. 289, no. 5485, pp. 1734-1736, 2000.

[27] M. I. Hussein, "Reduced Bloch mode expansion for periodic media band structure calculations," Proceedings of the Royal Society A: Mathematical, Physical and Engineering Sciences, vol. 465, no. 2109, pp. 2825-2848, Sep. 2009.

[28] B. Dzidek, S. Bochereau, S. A. Johnson, V. Hayward, and M. J. Adams, "Why pens have rubbery grips," Proceedings of the National Academy of Sciences, vol. 114, no. 41, pp. 10864-10869, 2017. 